\title{
SPATIAL INHOMOGENEITY OF PERIODIC AND CHAOTIC ATTRACTORS OF A DRIVEN DAMPED NONLINEAR SCHRÖDINGER EQUATION
}

\author{
A. ZUBRZYCKI \\ Department of Physics, Technical University \\ Malczewskiego 29, 26-600 Radom, Poland
}

(Received March 30, 1995; in final form July 20, 1995)

\begin{abstract}
The driven damped nonlinear Schrödinger equation is solved numerically and different attractors - periodic and chaotic ones - are obtained for different ranges of the forcing field amplitude. It is shown that these attractors are in both cases spatially nonuniform in time. Spatial inhomogeneity of the chaotic attractor is investigated by the estimation of the correlation dimension at different space points.
\end{abstract}

PACS numbers: 05.45.+b

It has been known for some time that completely integrable soliton systems perturbed by driving force and damping may exhibit a chaotic behaviour. The examples are the sine-Gordon equation [1] and the nonlinear Schrödinger equation $[2,3]$. In this paper the driven damped nonlinear Schrödinger equation of the same form as in Ref. [3] is considered

$$
q_{t}=\mathrm{i} q_{x x}+2 \mathrm{i}|q|^{2} q-\gamma q-\varepsilon \mathrm{e}^{\mathrm{i} \omega t} .
$$

The forcing field is characterized here by its amplitude $\varepsilon$ and frequency $\omega(>0)$ while the dissipation is given by $\gamma(>0)$. When $\varepsilon$ and $\gamma$ are both equal to zero, Eq. (1) reduces to the pure completely integrable nonlinear Schrödinger equation. The numerical simulation performed by Bekki and Nozaki [3] have shown that with increasing amplitude $\varepsilon$ of the forcing field ( $\gamma$ and $\omega$ were kept fixed) a solution of Eq. (1) undergoes a period doubling bifurcations from a coherent soliton to a chaotic solitary wave. In their work a time behaviour of the solution only at the centre of the system was examined. The present paper is focused on the investigation of the solution of Eq. (1) at different space locations. It is shown that the attractors reconstructed from the time series taken from diffcrent space locations are generally different.

Equation (1) was solved numerically in a domain $-L \leq x \leq L(L=8.1 \pi)$ for two types of boundary conditions: periodic and pinning ones. $A$ s there is no 
qualitative difference between solutions obtained for these two types of boundary conditions, most of the results shown below (cxcept Fig. 6b) are related to periodic boundary conditions. Equation (1) was solved by means of a pseudo-spectral method [4] in space and the third order Adams-Bashforth-Moulton predictor-corrector method in time (with a fixed time step 0.01). As an initial condition, a single static soliton

$$
q(x, 0)=2 \eta \operatorname{sech}(2 \eta x) \exp (-\mathrm{i} \pi / 2)
$$

with $\eta=0.3$ was taken. The calculations were carried out for fixed $\gamma(=0.1)$ and $\omega(=1.0)$, and $\varepsilon$ ranged from 0.1 to 0.15 . For a spatial discretization a lattice of 128 evenly spaced grid points was used and they are labelled from -64 to 63 so that the center of the static soliton is at the grid point number 0 . The soliton is static in the sense that it does not move along the $x$ axis and only its amplitude oscillates.

Firstly, in order to distinguish periodic attractors from chaotic ones the largest Lyapunov exponent was determined as a function of $\varepsilon$. It was derived by the method of Benettin et al. [5] from a solution of the equation tangent to Eq. (1)

$$
\psi_{t}=\mathrm{i} \psi_{x x}+2 \mathrm{i}\left(2|q|^{2} \psi+q^{2} \psi^{*}\right)-\gamma \psi
$$

where $\psi$ is a complex conjugate of $\psi^{*}$. The transition to a chaotic state takes place for $\varepsilon$ greater than about 0.148 as the largest Lyapunov exponent is positive above this value. Figure 1 depicts the largest Lyapunov exponent vs. $\varepsilon$ in the

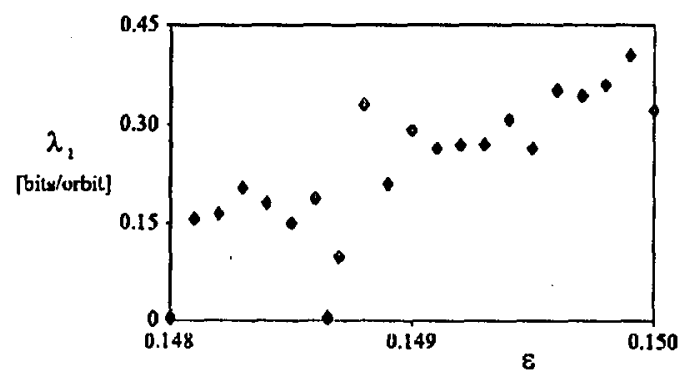

Fig. 1. The largest Lyapunov exponent as a function of the force field amplitude $\varepsilon$.

chaotic state. It is expressed in bits per orbit, where the mean orbital period is about 7.7 time units. The Lyapunov exponent was calculated with an equidistant step size $\Delta \varepsilon=0.0001$ within the given interval. Its zero value is also depicted for $\varepsilon=0.1486495$ for which a window of period- 6 solution exists.

In order to examine a space dependence of the solution of Eq. (1) in a periodic state, the attractor obtained for $\varepsilon=0.13$ was chosen. The results are shown in Figs. 2-4. Figures 2-4a (2a, 3a, 4a) depict phase portraits $|q|\left(t_{i}\right)$ vs. $|q|\left(t_{i}+\tau\right)$ reconstructed from the time series taken at three different points of the space; $t_{i}=i \Delta t$ is a multiple of time between successive data $(\Delta t=0.02)$ and $\tau$ is a delay time [6] $(\tau=1.5)$. Figures $2-4 b, c(2 b, c, 3 b, c, 4 b, c)$ depict time wave forms, and corresponding spectra of the amplitude of $q$ at these three points. It 

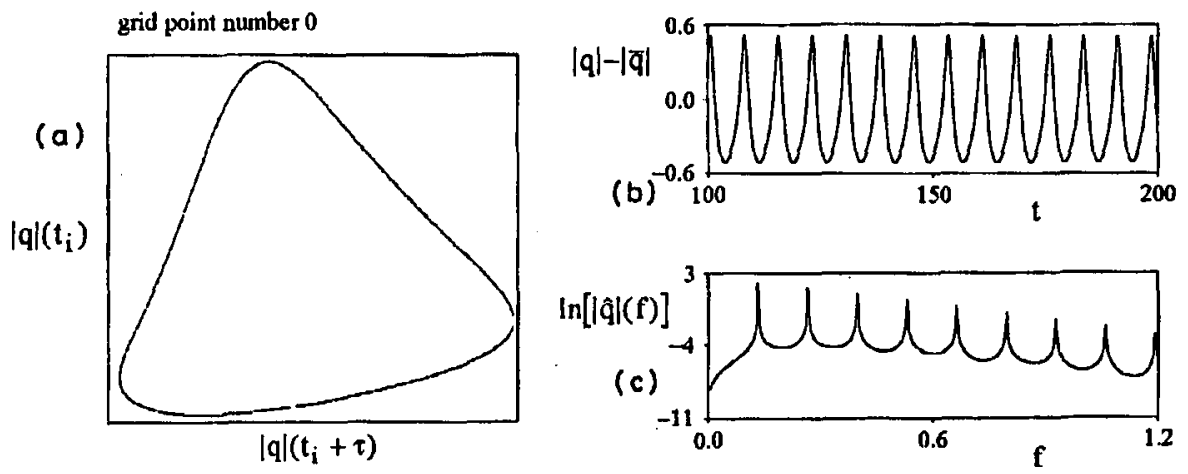

Fig. 2. Amplitude of the post-transient solution of Eq. (1) at the centre of the system, grid point number 0 , for $\varepsilon=0.13$. (a) Two-dimensional phase portraits $|q|\left(t_{i}\right)$ vs. $|q|\left(t_{i}+\tau\right)$ reconstructed from a time series. Time between successive data is $\Delta t=0.02$, $t_{i}=i \Delta t$, and a delay time $\tau=1.5$. (b) Time wave form of $|q|$. (c) Spectrum of $|q|$.

Fig. 3

(a)

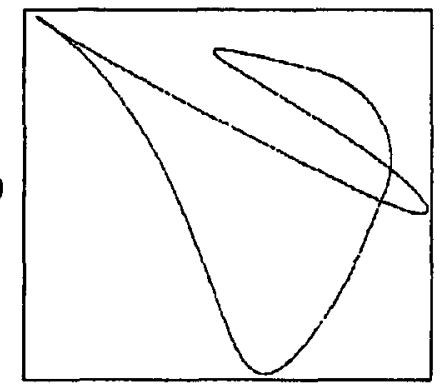

$|q|\left(t_{i}+\tau\right)$
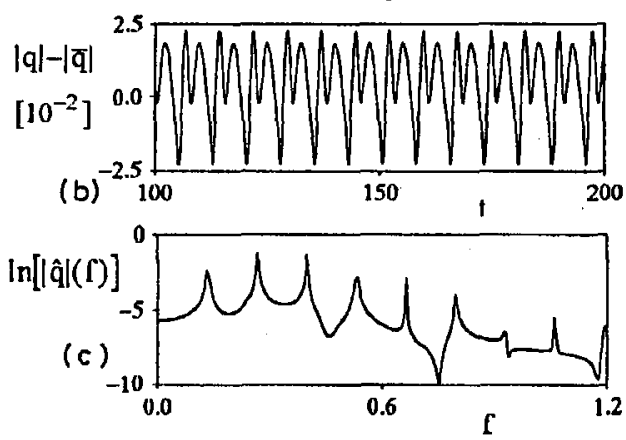

Fig. 4

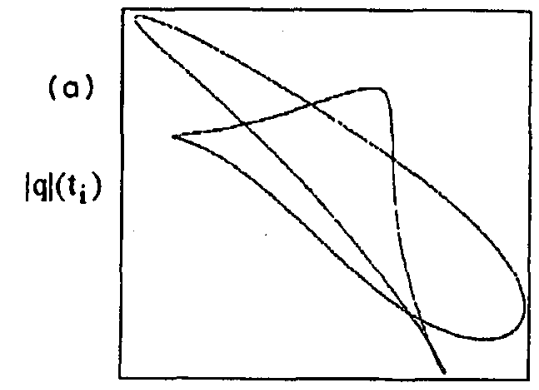

$|\mathrm{q}|\left(\mathrm{t}_{\mathrm{i}}+\tau\right)$
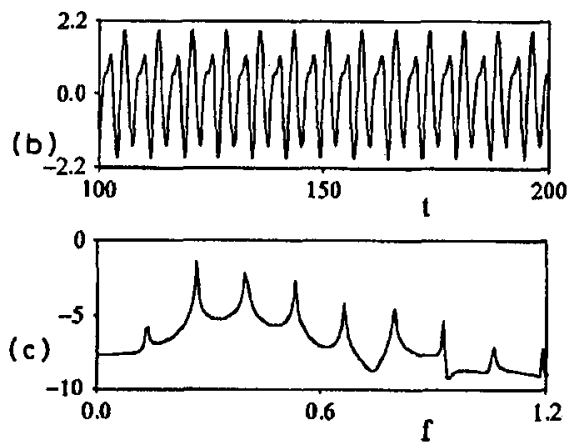

Fig. 3. Amplitude of the post-transient solution of Eq. (1) for $\varepsilon=0.13$ at grid point number 32. (a), (b), (c) the same as in Fig. 2.

Fig. 4. Amplitude of the post-transient solution of Eq. (1) at grid point number 63 for $\varepsilon=0.13$. (a), (b), (c) the same as in Fig. 2 . 
is seen that at the centre of the system (grid point number 0 ) we have harmonic oscillations with a fundamental frequency $\omega^{\prime} \approx 0.82$ smaller than the frequency of the forcing field $\omega=1.0$. Beyond the centre of the system, as we get closer to the boundaries, the oscillations become more complicated - amplitudes of some of the higher harmonics are relatively greater, while of the others - smaller. In particular, the first harmonic at $\omega^{\prime}$ gradually decreases and the second harmonic at $2 \omega^{\prime}$ becomes a dominant one as it is seen in Figs. 3c, $4 c$ for grid points number 32 and 63 , respectively. The results for negative grid points numbers are the same because of the symmetry of the attractor with regard to the system centre. The transient time, after which the oscillations at the particular location are periodic, becomes longer as a distance from the centre of the system increases. For higher $\varepsilon$ for which we have the period-doubling bifurcation process and the solution of Eq. (1) is a period-2 $(\varepsilon>0.141)$, period- $4(\varepsilon>0.146)$, etc. subharmonic, a spatial dependence of the solution is similar to that described above - the farther from the centre, the oscillations are more complicated and a contribution from higher harmonics is more visible. The spatial dependence of the time behaviour of the
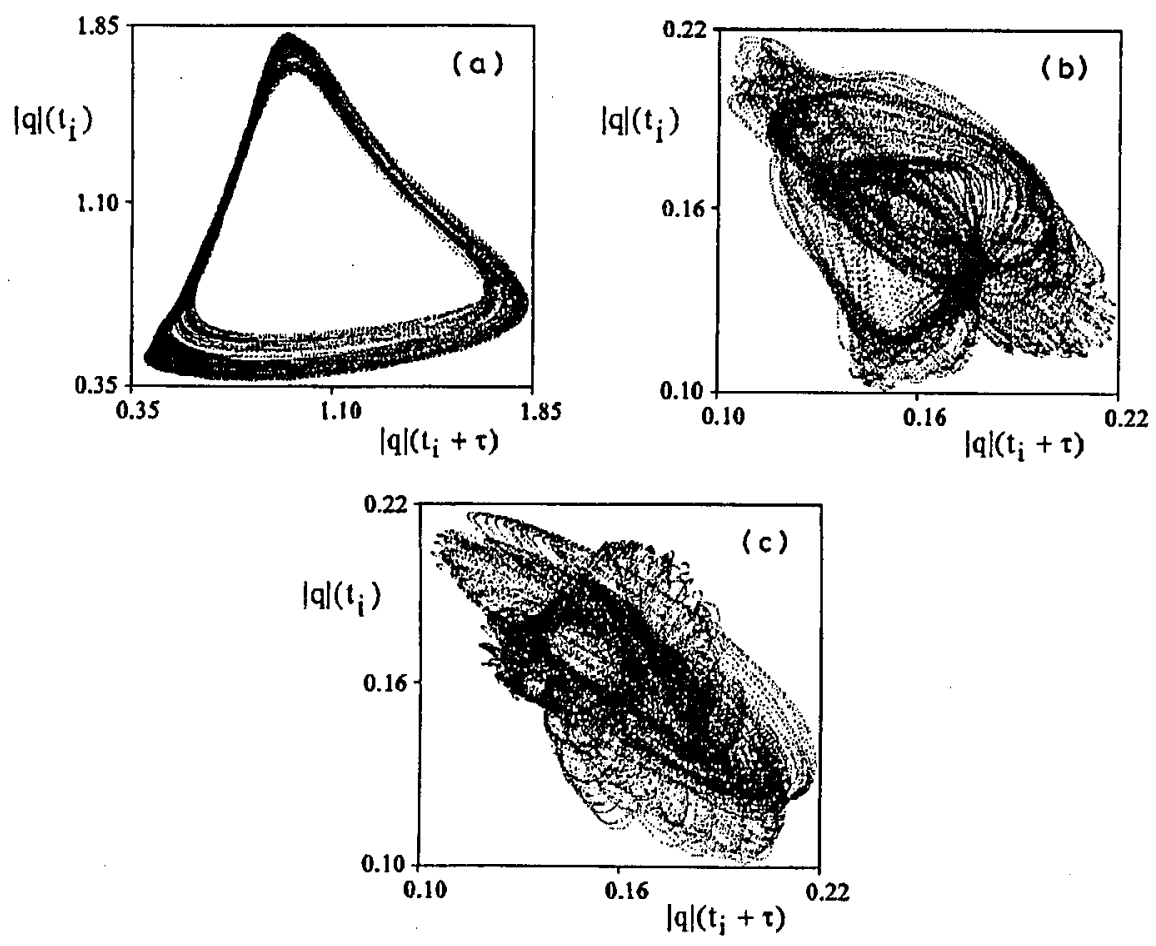

Fig. 5. Two-dimensional phase portraits $|q|\left(t_{i}\right)$ vs. $|q|\left(t_{i}+\tau\right)$ for $\varepsilon=0.149$, reconstructed from a time series taken at grid points number (a) 0 , (b) 32 , (c) 63 . Time between successive data is $\Delta t=0.02, t_{i}=i \Delta t$, and a delay time $\tau=1.5$. 
solution of Eq. (1) in the chaotic state will be presented for $\varepsilon=0.149$. From a comparison of the phase portraits $|q|\left(t_{i}\right)$ vs. $|q|\left(t_{i}+\tau\right)$ in Fig. 5 , reconstructed from the time series taken at grid points number 0,32 , and 63 , one can see that the chaotic attractor for $\varepsilon=0.149$ is spatially nonuniform. This spatial inhomogeneity was also examined quantitatively by the estimation of the correlation dimension $D_{\mathrm{c}}$ as a function of position along the $x$ axis. A modified Grassberger-Procaccia algorithm [7] was used to calculate the correlation dimension. At first the set of $N$ vectors in $m$-dimensional space,

$$
X(i)=\left\{|q|\left(t_{i}\right),|q|\left(t_{i}+\tau\right), \ldots,|q|\left(t_{i}+(m-1) \tau\right)\right\},
$$

where $1 \leq i \leq N$, was constructed from the time series and $M<N$, the so-called reference vectors were randomly chosen. Then, about each reference vector, the radius $r(j)$ of the smallest $m$-dimensional sphere containing exactly $j$ vectors $(1 \leq$ $j \leq N$ ) was found. The correlation dimension emerges as the slope (within a certain scaling region) of $\ln (j)$ vs. $\ln [\langle r(j)\rangle]$, where the angle brackets indicate averaging over all reference vectors. A large number of tests was carried out for a suitable choice of algorithm parameters to obtain a good scaling region - a straight line over a wide range. Finally, the calculations were performed with the embedding dimension $m=40$, the sampling time $\Delta t=0.15$, and the delay time $\tau=0.6$. The total number of vectors was $N=10000$ and number of reference vectors $M=400$. The error, for these values of the parameters, is estimated to be about $5 \%$. The correlation dimension was calculated at every eight grid point and the results are shown in Fig. 6. Figure 6a was obtained for periodic boundary
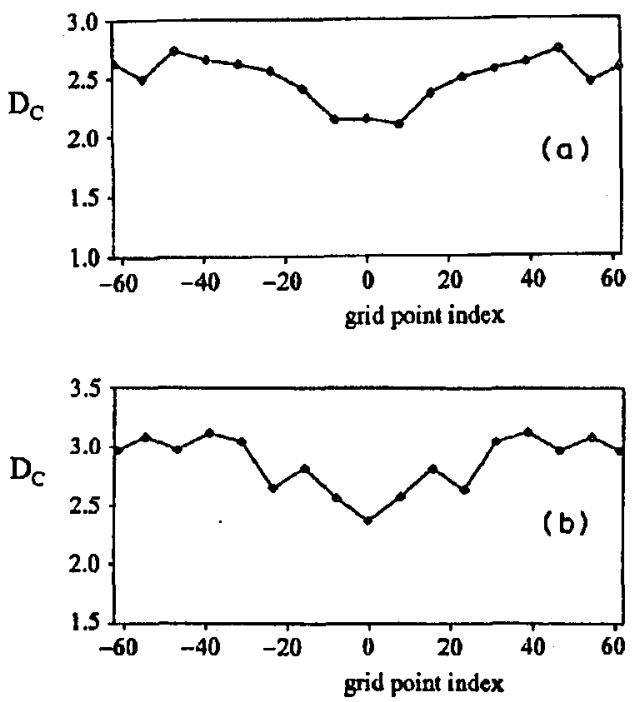

Fig. 6. Correlation dimension $D_{c}$ for $\varepsilon=0.149$ as a function of position along the $x$ axis (a) for periodic boundary conditions, (b) for pinning boundary conditions. 


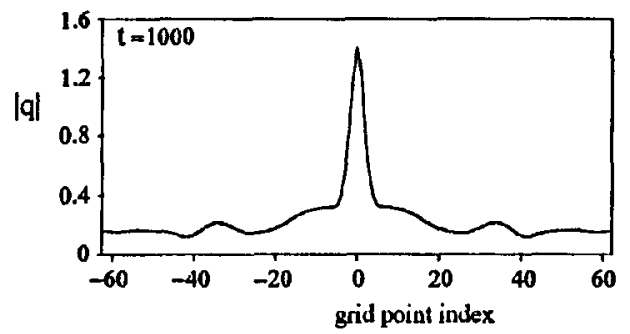

Fig. 7. Snap-sliot pattern of the amplitude of $q$ at the time $t=1000$.

conditions and Fig. $6 \mathrm{~b}$ for pinning boundary conditions. It is seen that for periodic boundary conditions the correlation dimension is rather small, ranging from about 2.1 to 2.7. It is the smallest and almost constant within the soliton (visible in Fig. 7), then it increases slowly with a growing distance from the centre of the system until it "jumps" down and up near the boundaries. Ilence, the attractor is spatially uniform within the soliton and the biggest inhomogeneities are related to the vicinity of the boundaries. For pinning boundary conditions the correlation dimension is a little larger, ranging from about 2.3 to 3.1 . It is again the smallest at the centre of the system, but there is no homogeneous region within the soliton. The attractors are in both cases symmetric with regard to the system centre. It should be added that the results are qualitatively similar for every other chaotic attractor that has been examined.

I would like to thank Professor Andrzej Sukiennicki for suggesting this problem to me and his helpful interest in the course of this work.

\section{References}

[1] J.C. Eilbeck, P.S. Lomdahl, A.C. Newell, Phys. Rev. Lett. A 87, 1 (1981); K. Nozaki, Phys. Rev. Lelt. 49, 1883 (1982).

[2] K. Nozaki, N. Bekki, Phys. Rev. Lett. 50, 1226 (1983); K. Nozaki, N. Bekki, Phys. Rev. Lett. A 102, 383 (1984).

[3] N. Bekki, K. Nozaki, Dynamical Problems in Soliton Systems 30, 268 (1985).

[4] B. Fornberg, G.B. Whitham, Phil. Trans. Roy. Soc. 289, 373 (1978).

[5] G. Benettin, L. Galgani, A. Giorgilli, J.-M. Strelcyn, Meccanica 15, 21 (1980).

[6] F. Takens, Lecture Notes in Mathemalics, Eds. D.A. Rand, L.-S. Young, Vol. 898, Springer, Berlin 1981, p. 366.

[7] J.W. Havstad, C.L. Ehlers, Phys. Rev. A 39, 845 (1989). 